\title{
Retinal lateral inhibition: an important mechanism for sharp-vision and color discrimination with high contrast sensitivity
}

Keywords: visual, retinal, lateral inhibition, horizontal cells, contrast sensitivity, color-vision, sharp-vision

Abbrevations: LI, lateral inhibition; RLI, retinal LI; HC, horizontal cell; GC, ganglion cell

\section{Editorial}

In this editorial, I aimed to give brief information about retinal lateral inhibition (RLI), an important visual phenomenon. Lateral inhibition (LI) was first discovered in horseshoe crabs and cats. ${ }^{1,2}$ However, its mechanism was first defined by Baylor et $\mathrm{al}^{3}$ In neurobiology, lateral inhibition (LI) or lateral antagonism is defined as the inhibition of the neuron's response to a stimulus via the excitation of the neighboring neuron. ${ }^{4-10}$ LI prevents the spread of action potentials from the stimulated neurons to the lateral neighboring neurons. Cells using LI have existed mainly in the cerebral cortex and thalamus. LI has been observed in the retina and lateral geniculate nuclei of the animals in experimental studies. Although LI has been identified primarily in the processings in visual sensation, it also occurs during sensory procedures such as touch, hearing, and smell. ${ }^{4-10}$ Retinal lateral inhibition (RLI) is also known as contrast encoder. RLI creates a stimulation contrast allowing increased sensory perception and enhances the contrast between the center and the periphery in a stimulated region. If activated at the same time, neighboring photoreceptors react less, although they are activated alone. Thus, when fewer neighboring neurons are stimulated, a neuron reacts more strongly. RLI is that the rod and cone photoreceptors in the perception zone interfere with each other to be active, inhibiting the response to central illumination by an increase in environmental illumination. ${ }^{1-10}$ When a certain type of cone is stimulated at a point, the same cone sends an inhibitory signal to the adjacent cone carrying the same photosensitive pigment to it with the help of horizontal cells (HCs). RLI is the main mechanism for achieving high visual acuity, sharpening the sensory location and color discrimination, which is involved in the transmission of contrasting edges in the visual image and increasing the contrasting sharpness. ${ }^{3,7-10}$

After the light beam comes to the retina via crossing the cornea, the pupil and the lens, it then bypasses ganglion cells (GCs), amacrine cells, bipolar cells, and HCs to reach the rod photoreceptors. The rods are stimulated by light and give a neural signal to stimulate the HCs. However, this stimulating signal will only be transmitted to the GCs by rod cells in the middle of the GC receiving area, because the HCs respond by sending an inhibitory signal to the neighboring rod photoreceptors. The central rod cells send light signals directly to bipolar cells transmitting the signal to the GCs. Amacrine cells also provide LI to bipolar cells and GCs for various visual calculations such as image sharpening. Lastly, visual inputs are sent to the thalamus and cerebral cortex. LI is directed by HCs in the vertebrate retina. The mutual synapse between cone cells and horizontal cells mediates negative feedback. RLI allows visual images to be transmitted to the central nervous system with appropriate visual contrast. Visual

\author{
Volume 9 Issue 6 - 2019 \\ Burak Turgut \\ Department of Ophthalmology, Faculty of Medicine, Onsekiz \\ Mart University, Turkey
}

Correspondence: Burak Turgut, Department of
Ophthalmology, Faculty of Medicine, Onsekiz Mart University,
Turkey, Tel: +90533 7/28389, Email burakturgut@comu.edu.tr

Received: November II, 2019 | Published: December 16, 2019

contrast is enhanced by LI in some amacrine cells. This prevents the excitation signal from spreading to the environment by dendrite and axonal branching. ${ }^{3,7-10}$

Information received from the environment is suppressed by the information received from the center. During your eyes closed, if you close the tip of the pen at the tip of your finger, it can be recognized the place in which the pressure is localized although the circumference of the ground collapses under the primary effect of pressure. RLI explains the Hering grid illusion, simultaneous contrast, and Mach bands., ${ }^{3,7-10}$ In the visual system, RLI improves the perception of the edges and increases the contrast. $\mathrm{HC}$ is primarily responsible for RLI. HC receives chemical synaptic inputs from photoreceptors and produces a feedback signal via the release of the neurotransmitter (the reciprocal synapse)..$^{3,7-10}$ In conclusion, RLI is a visual phenomenon performed by HCs and amacrine cells in the retina. It achieves high visual acuity, sharpening the sensory location and color discrimination, which is involved in the transmission of contrasting edges in the visual image and increasing the contrasting sharpness.

\section{Authorship contributions}

The named author meets the International Committee of Medical Journal Editors (ICMJE) criteria for authorship of this manuscript, take responsibility for the integrity of the work as a whole, and has given final approval to the version to be published.

\section{Acknowledgments}

None.

\section{Conflicts of interest}

The author declares that there is no conflict of interest regarding the publication of this paper.

\section{References}

1. Hartline HK, Ratliff F. Spatial summation of inhibitory influences in the eye of Limulus, and the mutual interaction of receptor units. J Gen Physiol. 1958;41(5):1049-1066. 
2. Kuffler SW. Discharge patterns and functional organization of the mammalian retina. J Neurophysiol. 1953;16(1):37-68.

3. Baylor DA, Fuortes MG, O’Bryan PM. Receptive fields of cones in the retina of the turtle. J Physiol. 1971;214(2):265-294.

4. Yantis S. Sensation and Perception. New York: Worth Publishers; 2014. p. 77.

5. Bakshi A, Ghosh K. A Neural Model of Attention and Feedback for Computing Perceived Brightness in Vision. In: Samui P, Roy SS, Balas VE, editors. Handbook of Neural Computation. London: Academic Press-Elsevier; 2017. p. 487-513.

6. Heller MA, Gentaz E. Psychology of Touch and Blindness. NewYork: Psychology Press; 2013. p. 20-21.
7. Levine $M W$. Levine \& Shefner's fundamentals of sensation and perception. 3rd ed. Oxford, England: Oxford University Press. 2006.

8. Tanaka M, Tachibana M. Independent control of reciprocal and lateral inhibition at the axon terminal of retinal bipolar cells. $J$ Physiol. 2013;591(16):3833-3851.

9. Kramer RH, Davenport CM. Lateral inhibition in the vertebrate retina: the case of the missing neurotransmitter. PLoS Biol. 2015;13(12):e1002322.

10. Roska B, Nemeth E, Orzo L, et al. Three levels of lateral inhibition: A space-time study of the retina of the tiger salamander. J Neurosci. 2000;20(5):1941-1951. 